\title{
Conductance Switching of Alkane Molecules by Gauche Conformation on Au(111)
}

\author{
Katsunori Tagami* \\ Department of Molecular Engineering, Graduate School of Bioscience and Biotechnology, \\ Tokyo Institute of Technology, 4259 Nagatsuta-cho, \\ Midori-ku, Yokohama-shi, Kanagawa 226-8501, Japan, and \\ Department of Nano-Science and Nano-engineering, \\ School of Science and Engineering, Waseda University, \\ 513 Wasedatsurumaki-cho, Shinjuku-ku, Tokyo 162-0041, Japan \\ Masaru Tsukada \\ Department of Nano-Science and Nano-engineering, \\ School of Science and Engineering, Waseda University, \\ 513 Wasedatsurumaki-cho, Shinjuku-ku, Tokyo 162-0041, Japan \\ (Received 30 June 2004; Accepted 2 July 2004; Published 8 July 2004)
}

\begin{abstract}
We investigated theoretically the effects of molecular rotation on the STM tunneling conductance for three types of $\sigma$-bonded molecules, i.e., (a) bicyclo[2,2,2] octylmethylthiolate (BCO), (b) cyclohexylmethylthiolate (cHex), and (c) 1-pentanethiolate (C5), which are anchored to the $\mathrm{Au}(111)$ surface by $\mathrm{Au}-\mathrm{S}$ bond. In the case of $\mathrm{BCO}$ and $\mathrm{cHex}$, the conductance does not change when the molecules transform from the most stable to metastable configurations. In contrast, in the case of $\mathrm{C} 5$, the conductance is found to decrease markedly, which means that transformation from the all-trans to gauche conformation can work as a switch between ON and OFF states. This finding would give a possible mechanism of the conductance switching recently observed in the STM images for the C5 molecules inserted into the BCO SAM matrix. [DOI: 10.1380/ejssnt.2004.186]
\end{abstract}

Keywords: Conductance switching, Green's functions methods, Computer simulations, Electrical transport, Tunneling, Alkanes, Nano-scale imaging

\section{INTRODUCTION}

Inserted molecules into the self-assembled monolayer (SAM) matrices formed on the $\mathrm{Au}(111)$ surface have been observed to blink frequently in the scanning tunneling microscopy (STM) images. It means that the adsorbed molecule has two conducting states, ON and OFF states. So far a few mechanisms for this conductance switching have been proposed, i.e., (1) molecular motion including tilting [1-3], (2) fluctuation of Au-S bond which anchors the molecule to the $\mathrm{Au}(111)$ surface [4], (3) conformational change of the molecule by rotation $[5,6]$. In the case of phenylene-ethynylene oligomers, the third mechanism due to the phenyl ring rotation has been excluded by comparison of data from several molecules. In contrast, in the case of alkane molecules, this mechanism has not been eliminated. The authors in Ref. [6] considered that by its repulsive force the tip induces transformation of the alkane molecules from the ground-state all-trans conformation to the metastable gauche conformation. The latter structure itself has been observed in the dodecanethiolate SAM pushed by the tip [7], and in a variety of Langmuir-Blodgett and self-assembled films of long alkyl chains under high pressure [8-10]. In addition, a theoretical simulation of the hexadecanethiolate SAM showed that gauche defects were formed along the chain with increasing pressure [11]. However, it has not been made clear how the tunneling conductance of single alkanethiolate molecules inserted into the SAM matrix actually changes against the molecular rotation. Then, our purpose of the present paper is to clarify their relationship in detail by theoretical simulations.

*Corresponding author: tagami@cms.nano.waseda.ac.jp

\section{MODEL AND METHOD}

We consider three types of $\sigma$-bonded molecules with similar height, i.e., (a) bicyclo[2,2,2] octylmethylthiolate (BCO), (b) cyclohexylmethylthiolate (cHex), and (c) 1pentanethiolate (C5), which are anchored to the $\mathrm{Au}(111)$ surface by Au-S bonds ( see Fig. 1 ). For brevity, we fix all the structural parameters except the rotation around a certain $\mathrm{C}-\mathrm{C}$ bond normal to the surface. In addition, as a crude approximation, we neglect the effects of the surrounding SAM matrix with which the inserted molecules interact via van der Waals force $[3,12,13]$. Their atomic configurations are modeled in the following way. The lengths of $\mathrm{C}-\mathrm{H}, \mathrm{C}-\mathrm{C}$, and C-S bonds are fixed to 1.10, 1.54 , and $1.92 \AA$, respectively, and all the bond angles are fixed to $109.4^{\circ}$. The sulfur atom is located at the height of $2.55 \AA$ on top of a gold atom of the ideal $\mathrm{Au}(111)$ surface. These quantities are fixed even if we consider the rotation of these molecules around a certain $\mathrm{C}-\mathrm{C}$ bond perpendicular to the surface. Thus, in our model, the molecular

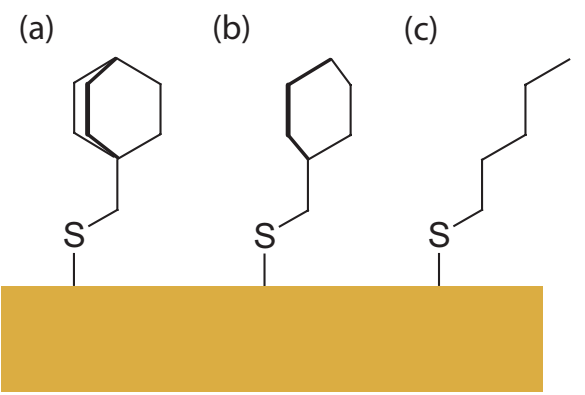

FIG. 1: Three types of $\sigma$-bonded molecules anchored to $\mathrm{Au}(111)$ surface by $\mathrm{Au}-\mathrm{S}$ bond. (a) BCO, (b) cHex, and (c) C5. 
height is assumed to be exactly the same, irrespective of the molecular species and their rotation angles.

Here we should comment on the simplification in our model. In realistic situations, the bond lengths and bond angles would change according to their bonding environment, and would deviate from their typical values of the $\sigma$ bonds $(1.54 \AA)$ and the $\mathrm{sp}^{3}$ bond angle $\left(109.4^{\circ}\right)$, respectively. Furthermore, the sulfur atom may not be just on top of an Au atom [14-19]. Thus, in principle, all of these factors should be taken into account in studying the energetics and transport properties of these molecules. However, a physically transparent analysis would be difficult, if we treat all the relevant parameters at the same time. Then, as a first step, we will model the system with all the structural parameters fixed except the rotation in consideration.

In discussing the energetics of the molecules adsorbed on the gold surfaces, we adopt the cluster model which is composed of the molecule and a fragment of the ideal $\mathrm{Au}(111)$ surface. The latter part is represented by 19 $\mathrm{Au}$ atoms, which mimics an $\mathrm{Au}(111)$ thin film of two layers thickness. For example, in the case of $\mathrm{BCO}$ on $\mathrm{Au}(111), \mathrm{Au}_{19}-\mathrm{S}-\mathrm{CH}_{2}-\mathrm{C}_{8} \mathrm{H}_{13}$ molecule is considered as a cluster. The total energies for the three types of molecules at given rotation angles are obtained by ab-initio calculations based on the density-functional theory, assuming the B3LYP exchange-correlation functional [20, 21] with the LANL2DZ bases.

As for the tunneling conductance, we assume the periodic boundary conditions along the lateral directions whose dimensions are $14.1 \AA \times 16.3 \AA$. In each supercell, a single molecule is located on top of a gold atom on the ideal $\mathrm{Au}(111)$ surface with the $\mathrm{S}-\mathrm{Au}$ distance set to $2.55 \AA$. Here, this surface is assumed to have semi-infinite layers thickness, which is in contrast to the cluster calculations previously mentioned. In a similar manner, the tip is modeled as the ideal $\mathrm{Au}(111)$ electrode with semiinfinite layers thickness from which a single gold atom is protruded. The tip apex atom is located at $6.0 \AA$ on top of the highest hydrogen atom for all the three molecular species. Namely, even if the molecule is rotated and its top hydrogen atom moves around, the tip follows this motion with the $\mathrm{Au}-\mathrm{H}$ distance unchanged.

In order to treat numerically these infinitely extended systems, we adopt the extended-molecule approach [22, 23], coupled with Green's functions technique [24]. In this approach, not only the molecule but also a fraction of electrodes are considered as the extended molecule. The effects of the semi-infinite electrodes are embedded in the Hamiltonian of the extended molecule as the self-energy terms, which are calculated from the Green's functions. In our case, the top 3 layers (48 atoms) of the $\mathrm{Au}(111)$ surface, the molecules, the tip apex atom with 3 layers of the $\mathrm{Au}(111)$ atoms above it are assumed as the extended molecules. Thus, for example, in the case of BCO, $\mathrm{Au}_{48}-\mathrm{S}$ $\mathrm{CH}_{2}-\mathrm{C}_{8} \mathrm{H}_{13}-\mathrm{Au}-\mathrm{Au}_{48}$ is treated as the extended molecule.

Their electronic states are calculated by the selfconsistent tight-binding model based on the densityfunctional theory [25-31]. The Hamiltonian and overlap matrices are expanded by the atomic orbital sets, i.e., 1s orbital for hydrogen atoms, $2 \mathrm{~s}$ and $2 \mathrm{p}$ orbitals for carbon atoms, $3 \mathrm{~s}$ and $3 \mathrm{p}$ orbitals for sulfur atoms, and $5 \mathrm{~d}$ and 6 s orbitals for gold atoms. The onsite levels, and

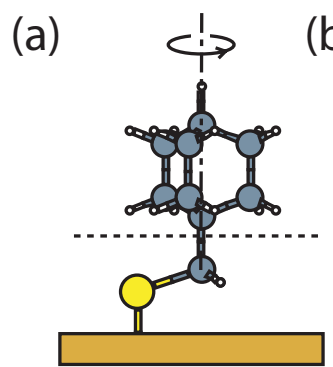

(b)

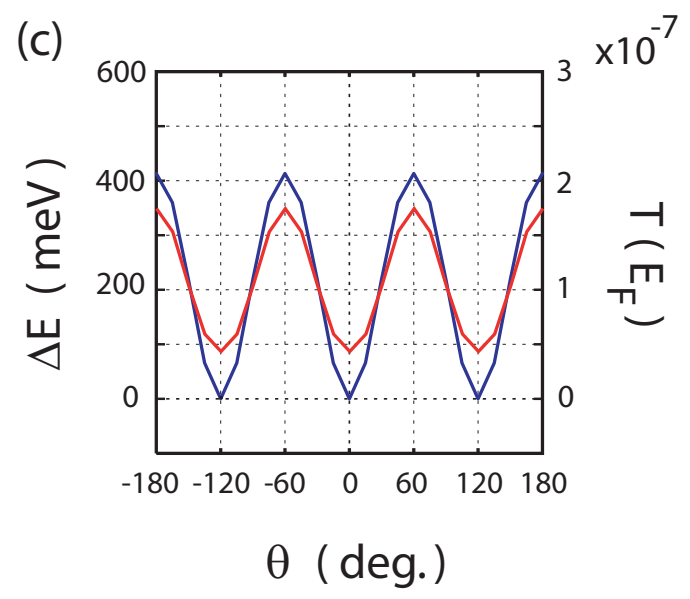

FIG. 2: (a),(b) Definition of rotation angle $\theta$ for BCO. (c) Energy curve (blue) and transmission probability curve (red) plotted against $\theta$.

the two-center hopping and overlap integrals are obtained from the norm-conserving atomic pseudo-potentials and the corresponding pseudo-wavefunctions, in a similar way to those reported in Ref. [25].

The charge distribution inside the gold surface with adsorbants and that inside the tip are obtained separately in a self-consistent manner. Note that since the tip is not very close to the surface, the charge redistribution between the tip and the adsorbants are not considered in this work. This kind of treatment is frequently adopted in the analyses or simulations of the STM images. Once the charge distribution is determined, the transmission amplitude $T\left(E_{F}\right)=G\left(E_{F}\right) / G_{0}$ between the tip and the molecule adsorbed on the $\mathrm{Au}(111)$ surface is calculated by the Landauer formula [26-31]. Here $G\left(E_{F}\right)$ and $G_{0}$ denote the conductance and quantum conductance, respectively, and $E_{F}$ denotes the Fermi level of the Au electrode determined beforehand from the band-structure calculation.

\section{RESULTS AND DISCUSSION}

\section{A. Conductance and rotational barrier}

Figure 2 (a) illustrates a snapshot of the BCO molecule rotated around the dash-dotted line. The atoms above the dotted line are allowed to move, and the rotation angle $\theta$ is defined in Fig. 2(b). As a function of $\theta$, the total energy differences $\Delta E(\theta)=E_{t o t}(\theta)-E_{t o t}(0)$ ( blue curve ) and the transmission probabilities at the Fermi level $T\left(E_{F}\right)$ ( red curve ) are plotted in Fig. 2 (c). The most energetically favorable configurations are found at 
(a)

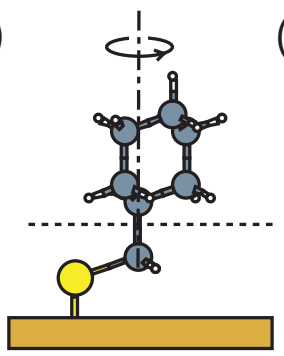

(b)

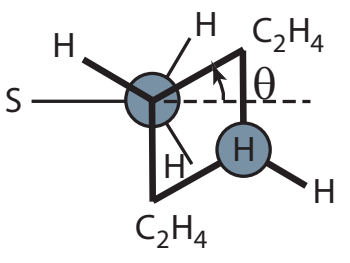

(c)

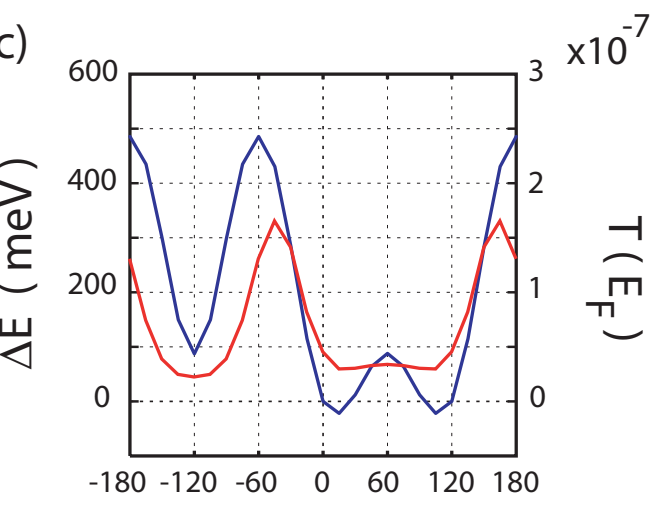

$\theta$ (deg.) (a)

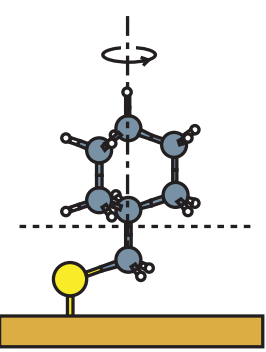

(b)

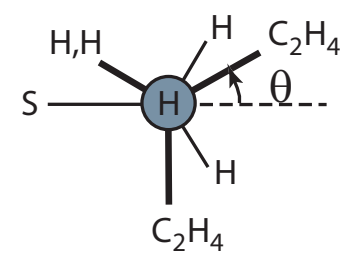

(c)

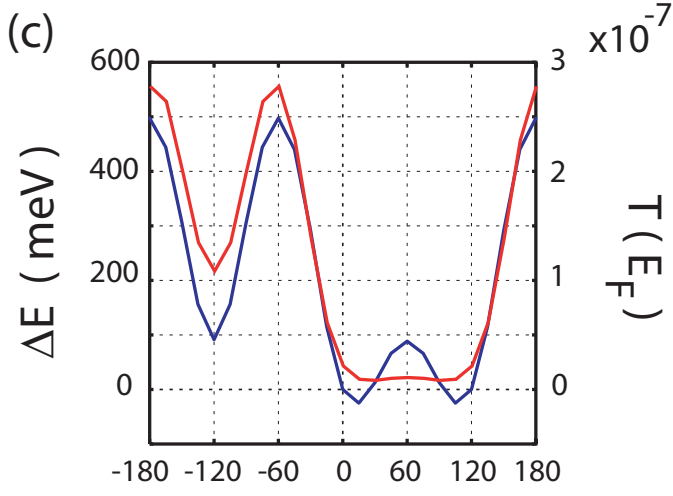

$\theta$ (deg.)
FIG. 3: (a),(b) Definition of rotation angle $\theta$ for cHex with chair conformation. c) Energy curve (blue) and transmission probability curve (red) plotted against $\theta$.

$\theta=0$ and $\pm 120^{\circ}$. Correspondingly, $T\left(E_{F}\right)$ shows a similar oscillating curve against $\theta$. Namely, $T\left(E_{F}\right)$ takes a minimal value, $4.4 \times 10^{-8}$ at $\theta=0$ and $\pm 120^{\circ}$, and takes a maximal value, $1.7 \times 10^{-7}$ at $\theta= \pm 60^{\circ}, \pm 180^{\circ}$. It means that the conductance can be changed by 4 times by rotating the molecule. However, as the rotational barrier between these local minima is estimated to be about 400 $\mathrm{meV}$, the transition from one local minimum to the other is considered to be suppressed at lower temperature.

Figure 3 (a) illustrates a snapshot of the cHex molecule with a chair conformation rotated around the dash-dotted line. Again, the atoms above the dotted line are allowed to move, and the rotation angle $\theta$ is defined in Fig. 3(b). In a similar manner as in the case of BCO, Fig. 3(c) plots $\Delta E$ ( blue ) and $T\left(E_{F}\right)$ ( red ). The local minima on the energy curve are found at $\theta \sim 15^{\circ}, 105^{\circ}$, and $-120^{\circ}$. Correspondingly, $T\left(E_{F}\right)$ takes $2.9 \times 10^{-8}, 2.9 \times 10^{-8}, 2.1 \times 10^{-8}$. However, in contrast to the case of $\mathrm{BCO}$, the positions of the minimum and maximum of $T\left(E_{F}\right)$ do not agree with those of $\Delta E(\theta)$. Namely, they are found at $\theta=-$ $120^{\circ}$ with $T\left(E_{F}\right)=2.1 \times 10^{-8}$, and at $\theta \sim 165^{\circ}\left(-45^{\circ}\right)$ with $T\left(E_{F}\right)=1.6 \times 10^{-7}$, respectively.

Note here that the rotational barrier between the two local minima at $\theta \sim 15^{\circ}$ and $105^{\circ}$ is only about $110 \mathrm{meV}$, although the barrier for transition to the third local minimum exceeds $500 \mathrm{meV}$. Thus, transition between the first two local minima would occur more frequently than in the case of BCO. However, $T\left(E_{F}\right)$ does not change its value upon this kind of molecular rotation.

These features are also found in the case of the cHex molecule with a boat conformation, as illustrated in Fig. 4. While the shape of the energy curve does not change markedly from that of the cHex with the chair conforma-
FIG. 4: (a),(b) Definition of rotation angle $\theta$ for cHex with boat conformation. (c) Energy curve (blue) and transmission probability curve (red) plotted against $\theta$.

tion, the transmission probabilities change significantly. The minimum and maximum of $T\left(E_{F}\right)$ appear at $\theta \sim 30^{\circ}$ $\left(90^{\circ}\right)$ with $T\left(E_{F}\right)=7.8 \times 10^{-9}$ and at $\theta \sim 180^{\circ}\left(-60^{\circ}\right)$ with $T\left(E_{F}\right)=2.8 \times 10^{-7}$, respectively. At the positions of the local minima of $\Delta E\left(\theta=15^{\circ}, 105^{\circ}\right.$, and $\left.-120^{\circ}\right), T\left(E_{F}\right)$ takes values of $8.8 \times 10^{-9}, 8.8 \times 10^{-9}, 1.1 \times 10^{-7}$, respectively. Also in this case, the difference in the conductance is not observed between the two equivalent local minima at $\theta=15^{\circ}$ and $105^{\circ}$.

Finally, we consider the rotation of the C5 molecules. Figure 5 (a) illustrates a snapshot of the C5 molecule rotated around the dash-dotted line. The rotation angle $\theta$ is defined in Fig. 5(b). Figure $5(\mathrm{c})$ plots $\Delta E$ ( blue ) and $T\left(E_{F}\right)$ ( red ). $\Delta E(\theta)$ has local minima at $\theta=0^{\circ}$, $\sim \pm 105^{\circ}$. The rotational barrier from $\theta=0$ to $\pm 105^{\circ}$ is only about $148 \mathrm{meV}$. Thus, the transition between these two local minima would be possible by the thermal fluctuation, the force by the tip, and so on. To be attractive, $T\left(E_{F}\right)$ takes different values at these two conformations. Namely, $T\left(E_{F}\right)$ takes $6.8 \times 10^{-8}$ and $3.0 \times 10^{-8}$, respectively, for the former and latter conformation. It means that transformation from all-trans to gauche conformation causes a dramatic change in the conductance through the C5 molecule. The ON/OFF switching ratio in the absolute value of the conductance is about 2.3.

The situation does not change even if the gauche defect is introduced at the tail of the molecule as illustrated in Fig. 6(a). The atoms above the dotted line are allowed to move, and the rotation angle $\theta$ is defined in Fig. 6(b). Figure $6(\mathrm{c})$ plots $\Delta E$ ( blue ) and $T\left(E_{F}\right)$ ( red ). While the energy curve does not change significantly from that seen in Fig. 5(c), the transmission probabilities change 
(a)

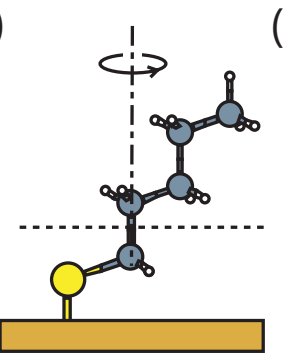

(b)

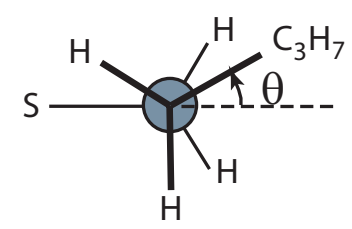

(c)

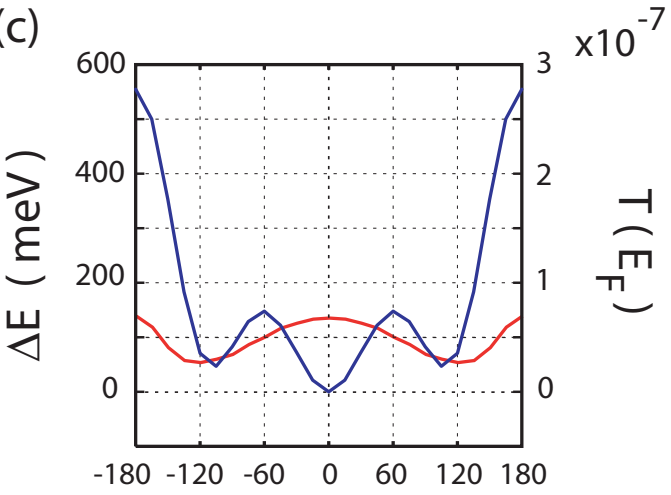

$\theta$ ( deg.) (a)

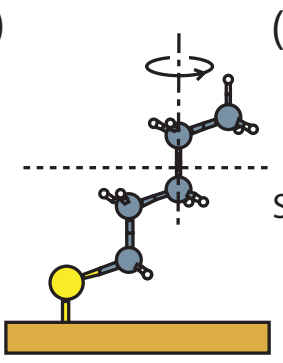

(b)

(c)

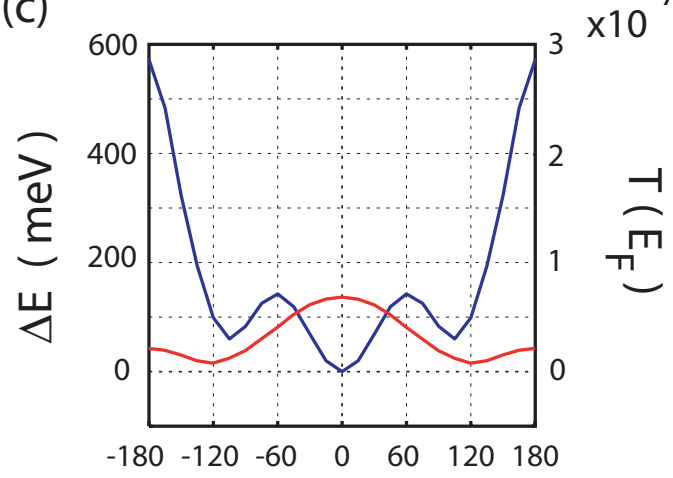

$\theta$ (deg.)
FIG. 5: (a),(b) Definition of rotation angle $\theta$ for C5. (c) Energy curve (blue) and transmission probability curve (red) plotted against $\theta$.

dramatically. At the local minima of $\Delta E$ at $\theta=0, \pm 105^{\circ}$, $T\left(E_{F}\right)$ takes values of $6.8 \times 10^{-8}, 1.2 \times 10^{-8}$, respectively. Thus, transformation to gauche conformation results in a conductance switching whose $\mathrm{ON} / \mathrm{OFF}$ ratio amounts to about 5.7 .

\section{B. Switching in STM images}

Then, we wonder if the C5 and BCO molecules coexist on the $\mathrm{Au}(111)$ surface. In this case, how do these adsorbants look in the STM images? As mentioned previously, both $\mathrm{BCO}$ and $\mathrm{C} 5$ molecules take the most stable configurations at $\theta=0$. At this time their transmission amplitudes $T\left(E_{F}\right)$ are $4.4 \times 10^{-8}$ and $6.8 \times 10^{-8}$, for the corresponding molecules. Thus, when the tip is fixed at the same height, the C5 molecule is expected to be seen brighter than the BCO molecule in the STM images. However, if the C5 molecule changes its conformation to the other local minima, its transmission probability $T\left(E_{F}\right)$ is reduced to $3.0 \times 10^{-8}$ ( see Fig.5 ) or $1.2 \times 10^{-8}$ ( see Fig.6 ). In either case, $T\left(E_{F}\right)$ takes a lower value than that of the $\mathrm{BCO}, 4.4 \times 10^{-8}$. It means that the $\mathrm{C} 5$ molecule is now expected to be seen darker than the BCO molecule. When this kind of conformational change occurs frequently, the blinking of the C5 molecule should be observed in the STM images. Indeed, in a recent STM experiment, the C5 molecules inserted into the BCO SAM matrix were observed to blink [6].

At a first sight it may seem strange that the STM images are so sensitive to the internal structure of the alkanethiolate molecule. However, according to a first-
FIG. 6: (a),(b) Definition of rotation angle $\theta$ for C5. (c) Energy curve (blue) and transmission probability curve (red) plotted against $\theta$.

principles calculation which explains well the STM images of heptanethiol SAM [13], the STM visualizes the LDOS at the tail of the alkyl chains, and not the wavefunctions of the $\mathrm{Au}$ or $\mathrm{S}$ atom. It means that the tunneling current is sensitive to the internal structure of the adsorbants through which the tunneling electron passes. Therefore, it should be possible that the conformational change of the alkyl chain is visualized in the STM images. As denoted previously, however, we ignored van der Waals interaction between the alkyl chain and the surrounding BCO SAM matrix, which may limit the possible range of molecular rotation. In order to investigate such an additional factor on the STM images, theoretical simulations should be necessary in which more than a few hundred atoms are concerned.

\section{CONCLUSION}

In conclusion, we have shown that in the case of BCO and cHex, the tunneling conductance does not change when the molecules transform from the most stable to metastable configurations. In contrast, in the case of C5, the conductance is found to decrease markedly, which means that transformation from the all-trans to gauche conformation can work as a switch between ON and OFF states. This finding would give a possible mechanism of the conductance switching recently observed in the STM images for the C5 molecules inserted into the BCO SAM matrix. 


\section{Acknowledgments}

We thank Mr. M. Suzuki, Mr. S. Fujii, and Prof. M. Fujihira for fruitful discussion on STM images of various kind of molecules. This work was supported in part by the Ministry of Education, Culture, Sports, Science and Technology, Grant-in-Aid for Creative Scientific Research "Devices on molecular and DNA levels". The numerical calculations were performed on SR8000 at the Computer Center at the University of Tokyo.
[1] Z. J. Donhauser, B. A. Mantooth, T. P. Pearl, K. F. Kelly, S. U. Nanayakkara, and P. S. Weiss, Jpn. J. Appl. Phys. 41, 4871 (2002).

[2] Z. J. Donhauser, B. A. Mantooth, K. F. Kelly, L. A. Bumm, J. D. Monnell. J. J. Stapleton, D. W. Jr. Price, A. M. Rawlett, D. L. Allara, J. M. Tour, and P. S. Weiss, Science 291, 2303 (2001).

[3] S. Wakamatsu, S. Fujii, U. Akiba, and M. Fujihira, Nanotechnology 14, 258 (2003).

[4] G. K. Ramachandran, T. J. Hopson, A. M. Rawlett, L. A. Nagahara, A. Primak, and S. M. Lindsay, Science 300, 1413 (2003).

[5] J. M. Seminario, P. A. Derosa, and J. L. Bastos, J. Am. Chem. Soc. 124, 10266 (2002).

[6] M. Suzuki, S. Fujii, S. Wakamatsu, U. Akiba, and M. Fujihira, Nanotechnology 15, S150 (2004).

[7] T. Ignor and B. G. Christopher, J. Phys. Chem. B 101, 5263 (1997).

[8] O. Berg and M. Klenerman, J. Appl. Phys. 90, 5070 (2001).

[9] F. Eisert, M. Gurka, A. Legant, M. Buck, and M. Grunze, Science 287, 468 (2000).

[10] Q. Du, X-. D. Xiao, D. Charych, F. Wolf, P. Frantz, Y. R. Chen, and M. Salmeron, Phys. Rev. B 51, 7456 (1995).

[11] J. I. Siepmann and I. R. McDonald, Phys. Rev. Lett. 70, 453 (1993).

[12] M. Hyun and C. K. Rhee, Bull. Korean Chem. Soc. 22, 213 (2001).

[13] C. Zeng, B. Li, B. Wang, H. Wang, K. Wang, J. Yang, J. G. Hou, and Q. Zhu, J. Chem. Phys. 117, 851 (2002).

[14] Y. Yourdshahyan and A. M. Rappe, J. Chem. Phys. 117, 825 (2002).

[15] Y. Akinaga, T. Nakajima, and K. Hirao, J. Chem. Phys.
114, 8555 (2001).

[16] M. C. Vargas, P. Giannozzi, A. Selloni, and G. Scoles, J. Phys. Chem. B 105, 9509 (2001).

[17] J. Gottschalck and B. Hammer, J. Chem. Phys. 116, 784 (2002).

[18] S. Franzen, Chem. Phys. Lett. 381, 315321 (2003).

[19] Y. Morikawa, Phys. Rev. B 63, 033405 (2001).

[20] A. D. Becke, J. Chem. Phys. 98, 5648 (1993).

[21] C. Lee, W. Yang, and R. G. Parr, Phys. Rev. B 37, 785 (1988).

[22] J. Taylor, H. Guo, and J. Wang, Phys. Rev. B 63, 245407 (2001).

[23] Y. Xue, S. Datta, and M. A. Ratner, J. Chem. Phys. 115, $4292(2001)$

[24] M. B. Nardelli, Phys. Rev. B 60, 7828 (1999).

[25] T. Frauenheim, G. Seifert, M. Elstner, T. Niehaus, C. Köhler, M. Amkreutz, M. Sternberg, Z. Hajnal, A. D. Carlo, and S. Suhai, J. Phys.: Cond. Matt. 14, 3015 (2002).

[26] K. Tagami, M. Tsukada, T. Matsumoto, and T. Kawai, Phys. Rev. B. 67, 245324 (2003).

[27] K. Tagami and M. Tsukada, Jpn. J. Appl. Phys. 42, 3606 (2003).

[28] K. Tagami and M. Tsukada, e-J. Surf. Sci. Nanotech. 1, 45 (2003).

[29] K. Tagami, M. Tsukada, Y. Wada, T. Iwasaki, and H. Nishide, J. Chem. Phys. 119, 7941 (2003).

[30] K. Tagami and M. Tsukada, J. Phys. Chem. B. 108, 644 (2004).

[31] K. Tagami, L. G. Wang, and M. Tsukada, NanoLetters 4, 209 (2004). 